\title{
Erythropoietin-induced upregulation of endothelial nitric oxide synthase but not vascular endothelial growth factor prevents musculocutaneous tissue from ischemic damage
}

\author{
Farid Rezaeian ${ }^{1,2}$, Reto Wettstein ${ }^{1}$, Jean-François Egger ${ }^{3}$, Freya Sandmann², Martin Rücker ${ }^{4}$, Mickaël Tobalem², \\ Brigitte Vollmar ${ }^{5}$, Michael D Menger ${ }^{6}$ and Yves Harder ${ }^{1,2}$
}

Recent findings have attested the protective effects of erythropoietin (EPO) in ischemically challenged organs. We therefore aimed at elaborating the underlying mechanism of EPO-mediated protection in musculocutaneous tissue undergoing persistent ischemia after acute injury. Mice were assigned to five experimental groups equipped with a randomly perfused flap fixed in a dorsal skinfold chamber, whereas the sixth group did not undergo flap preparation: EPO, L-Name, EPO and L-Name, EPO and bevacizumab, untreated flap, and nonischemic chamber (control). Intravital fluorescence microscopic analysis of microhemodynamics, apoptotic cell death, macromolecular leakage and angiogenesis was carried out over a 10-day period. Further, immunohistochemical analysis was used to study the protein expression of endothelial nitric oxide synthase (eNOS) and vascular endothelial growth factor (VEGF). Increased expression of eNOS in EPO-administered mice correlated with significant arteriolar dilation and thus increased blood flow resulting in a maintained functional capillary density (FCD) at day 10. In addition, EPO induced a VEGF upregulation, which was associated with newly formed capillaries. In addition, EPO was able to reduce ischemia-induced apoptotic cell death and finally to significantly reduce flap necrosis. In contrast, coadministration of L-Name abolished EPO-mediated tissue protection by abrogating the dilatory effect resulting in reduced FCD and tissue survival, without counteracting angiogenesis and apoptotic cell death, whereas additional administration of bevacizumab did not influence the beneficial effect of EPO on flap survival despite abrogating angiogenesis. Macromolecular leakage was found to be increased in all treatment groups. This study shows that EPO administration prevents musculocutaneous tissue from ischemic necrosis as a consequence of an eNOS-dependent arteriolar hyperperfusion maintaining capillary perfusion, thus representing a promising approach to pharmacologically protect ischemically challenged tissue.

Laboratory Investigation (2010) 90, 40-51; doi:10.1038/labinvest.2009.117; published online 9 November 2009

KEYWORDS: angiogenesis; critical ischemia; eNOS; EPO; flap surgery; microcirculation

Erythropoietin (EPO), a $30.4 \mathrm{kDa}$ glycoprotein, is the main regulator of erythropoiesis. ${ }^{1}$ It participates in the proliferation, differentiation and migration of erythroid progenitor cells. ${ }^{2}$ Accordingly, anemia ${ }^{3}$ and decreased tissue oxygen tension ${ }^{4}$ induce the production of EPO in the adult kidney. Recent findings revealed tissue-protective properties of EPO independent of its hematopoietic properties in a variety of organ systems suffering from ischemia, such as the heart, ${ }^{5}$ brain, ${ }^{6,7}$ liver, ${ }^{8}$ kidney ${ }^{9}$ and the striated muscle. ${ }^{10}$ EPO initiates a multitude of EPO receptor-dependent and -independent cellular pathways, including the induction of anti-apoptotic genes ${ }^{11}$ and anti-inflammatory molecules, ${ }^{12}$ the increase of vascular endothelial growth factor (VEGF), as well as the production of vasodilative molecules such as nitric

\footnotetext{
'Division of Plastic, Reconstructive \& Aesthetic Surgery, Geneva University Hospitals, Geneva, Switzerland; ${ }^{2}$ Faculty of Medicine, University of Geneva, Geneva, Switzerland; ${ }^{3}$ Department of Pathology, Geneva University Hospitals, Geneva, Switzerland; ${ }^{4}$ Department of Oral \& Maxillofacial Surgery, Hanover Medical School, Hanover, Germany; ${ }^{5}$ Institute for Experimental Surgery, University of Rostock, Rostock, Germany and ${ }^{6}$ Institute for Clinical \& Experimental Surgery, University of Saarland, Homburg/Saar, Germany

Correspondence: Dr Y Harder, MD, Division of Plastic, Reconstructive \& Aesthetic Surgery, Geneva University Hospitals (HUG), Rue Gabrielle-Perret-Gentil 4, Geneva $\mathrm{CH}-1211$, Switzerland.

E-mail: yvesharder@bluewin.ch

Received 28 October 2008; revised 31 August 2009; accepted 30 September 2009
} 
oxide (NO). ${ }^{13} \mathrm{NO}$ is an enzymatic product from L-arginine that mediates numerous biological actions through the activation of cyclic guanosine monophosphate, which inhibits intracellular calcium release and induces smooth muscle relaxation. ${ }^{14}$

As EPO and its receptor are also present in human skin, ${ }^{15}$ ischemic wound complications and tissue necrosis might be prevented by administration of EPO. Despite advances in surgical techniques, patients may still suffer from wound breakdown and ischemic necrosis. ${ }^{16,17}$ The associated morbidity and mortality represents a considerable health-care issue. ${ }^{18,19}$

The aim of this study was to evaluate the NO-dependent effect of systemic EPO administration starting before surgical induction of acute persistent ischemia in musculocutaneous tissue of mice. The role of microcirculatory, angiogenic and cellular mechanisms were analyzed by intravital microcopy and immunhistochemical work-up.

\section{MATERIALS AND METHODS Animals}

The experiments were conducted in accordance with the Swiss legislation on the protection of animals. The experimental protocol was approved by the Animal Ethics Committee of the Canton of Geneva, Switzerland. A total of 78 mice (C57BL/6; 12-24 weeks old; 24-28g body weight (bw); Zootechnie, Centre Médical Universitaire; Geneva, Switzerland) were used in the study. The animals were housed one per cage at room temperature of $22-24^{\circ} \mathrm{C}$ and at a relative humidity of $60-65 \%$ with a 12 -h day-night cycle. The animals had free access to standard pellet chow (Altromin, Lage, Germany) and tap water ad libitum.

\section{Anesthesia}

Both surgery and repetitive intravital fluorescence microscopy were performed under anesthesia by intraperitoneal (i.p.) injection of $0.1 \mathrm{ml}$ saline solution per $10 \mathrm{~g}$ bw of mixture of $90 \mathrm{mg}$ per $\mathrm{kg}$ bw ketamine hydrochloride (Ketavet; Parke Davis, Freiburg, Germany) and $25 \mathrm{mg}$ per $\mathrm{kg}$ bw dihydroxylidinothiazine hydrochloride (Rompun; Bayer, Zürich, Switzerland).

\section{Flap Preparation}

The dorsal skinfold chamber preparation, which takes $\sim 25 \mathrm{~min}$, was used as the control group. All other experimental groups were equipped with a skinfold chamber and a critically and randomly perfused musculocutaneous flap consisting of one layer of the skin, subcutis and striated muscle (panniculus carnosus). ${ }^{20}$ As previously described, the musculocutaneous tissue is subjected to persistent ischemia resulting in $\sim 50 \%$ necrosis if kept untreated. ${ }^{21}$ The preparation starts after depilation by mobilization of the musculocutaneous tissue (that is, induction of ischemia). The rectangular flap of $15 \times 11 \mathrm{~mm}$ remains attached to the animal only on its lateral border. Then it is fixed to the backside of the frame of the window chamber and sutured back to its previous position. Thereafter, the counterpart of the frame was mounted and the observation window is sealed with a coverglass, which replaces the previously removed musculocutaneous layer of the opposite layer of the skinfold, a fraction of skin that may be used for subsequent histological examination (time-point day 0 ). This preparation allows for repetitive microscopy of the same tissues. The animals tolerated the titanium chamber with a total weight of $\sim 3 \mathrm{~g}$ and the preparation well, as indicated by normal feeding and sleeping habits.

\section{Intravital Epifluorescence Microscopy}

For in vivo microscopic observation, animals were anesthetized and placed on a custom-made plexiglas frame. For intravascular contrast enhancement of the plasma they received intravenous (i.v.) retrobulbar injection of $0.1 \mathrm{ml} 5 \%$ fluorescein isothiocyanate (FITC)-labeled dextran (molecular weight $150000 ; 50 \mathrm{mg} / \mathrm{ml}$ saline; Sigma-Aldrich Chemie, Buchs, Switzerland). Subsequently, the animals were positioned under a Zeiss ${ }^{\circledR}$ Axiotech microscope (Zeiss, Feldbach, Switzerland) equipped with a $100-\mathrm{W}$ mercury lamp and a filter set for ultraviolet $(330-390 \mathrm{~nm}$ excitation/ $>430 \mathrm{~nm}$ emission wavelength) and blue (450-490 nm, >520 nm) light. Microscopic images were captured by a charge-coupled device video camera (Kappa CF 112, Zeiss) and transferred to a DVD recorder (Panasonic LQ-MD 800; Lucerne, Switzerland). All parameters were analyzed off-line using a computer-assisted image analysis system (Cap-Image; Zeintl Software, Heidelberg, Germany). ${ }^{22}$ The microscopic procedures for analysis of the microcirculation within the musculocutaneous tissue were performed at constant room temperature of $\sim 23^{\circ} \mathrm{C}$.

\section{Microcirculatory and Cellular Analysis}

Every observation started with scanning of the window in $\times 5$ magnification to determine the perfused as well as the non-perfused respectively the necrotic part of the tissue. Planimetrical evaluation was carried out thereafter and the percentage of the total area was calculated. Non-perfused tissue and necrosis are given as percentage of the total flap area.

All microcirculatory parameters were measured in perfused flap tissue. Easily identifiable branching patterns of second- or third-order arterioles accompanying collecting venules and capillary fields were selected. Video printouts of these branching patterns using the $\times 5$ and $\times 20$ magnification were made to relocate the same vessels for repetitive measurements during the 10-day observation period. (i) Arteriolar red blood cell (RBC) velocity $(\mathrm{mm} / \mathrm{s})$ was analyzed by a computer-assisted image analysis system using the line shift method, which is on the basis of the measurement of the shift $(\mathrm{mm})$ of an individual intravascular graylevel pattern over time (seconds). (ii) Arteriolar diameter $(\mu \mathrm{m})$ was measured perpendicularly to the vessel path. 
(iii) Volumetric blood flow $(\mathrm{pl} / \mathrm{s})$ was calculated in arterioles from RBC velocity and vessel cross-sectional area $\left(\pi \times r^{2}\right)$ according to the equation of Gross and Aroesty, that is, $Q=V \times \pi \times r^{2}$, assuming a cylindrical vessel shape. ${ }^{23}$ (iv) Functional capillary density (FCD: given in $\mathrm{cm} / \mathrm{cm}^{2}$ ) was defined as the length of all RBC-perfused capillaries per observation field. For assessment of FCD, the surface of the chamber window was subdivided into three horizontal areas of equal length resulting in a proximal zone directly adjacent to the lateral side where the tissue is attached to the animal, an intercalated central zone, and a distal zone most remote from the vascular inflow. (v) Apoptotic cells $\left(n / \mathrm{mm}^{2}\right)$ were identified by the characteristic nuclear condensation, fragmentation and margination with the aid of DNA-binding fluorochrome bisbenzimide H33342 (Sigma-Aldrich). Recordings were made with a $\times 100$ objective. (vi) The microcirculatory analysis further included the evaluation of the angiogenic response, which was analyzed by the density of all newly formed blood perfused microvessels (MVD). The vessel density was calculated as the total length of these newly formed vessels divided by the area of observation and is given in $\mathrm{cm} / \mathrm{cm}^{2}$. (vii) Macromolecular leakage as a parameter of microvascular permeability was assessed after an i.v. injection of FITC-labeled dextran 150 000, a macromolecular fluorescent dye. Gray levels in the tissue directly adjacent to the capillary vessel wall (E1), as well as in the marginal cell-free plasma of the vessel (E2) were determined densitometrically. Macromolecular extravasation (E) was then calculated as the ratio of E1/E2. ${ }^{24}$

\section{Immunohistochemical Analysis of eNOS and VEGF}

To assess the expression of endothelial nitric oxide synthase (eNOS) and VEGF using light microscopy, biopsies were snap-frozen and embedded in tissue-freezing medium (Jung; Leica Microsystems, Nussloch, Germany) or formalin fixed and embedded in paraffin. eNOS samples were cut in $5-\mu \mathrm{m}$ sections and fixed in $4{ }^{\circ} \mathrm{C}$ cold acetone for $5 \mathrm{~s}$ and in $4 \%$ formalin for $10 \mathrm{~min}$, whereas VEGF sections were stained using indirect immunoperoxidase techniques. eNOS sections were incubated with the polyclonal rabbit anti-mouse eNOS antibody (1:25; BD Biosciences, Heidelberg, Germany). VEGF sections were incubated at $4{ }^{\circ} \mathrm{C}$ overnight with a mouse monoclonal anti-mouse VEGF antibody (1:25; M7273, DakoCytomation, Hamburg, Germany) as primary antibody. A peroxidase-conjugated goat anti-rabbit IgG antibody was used as secondary antibody (1:200; Dianova, Hamburg, Germany) for both primary antibodies. 3,3'-Diaminobenzidine (DakoCytomation) was used as a chromogen. Sections were counterstained with Mayer's hemalaun. All sections were incubated with $3 \% \mathrm{H}_{2} \mathrm{O}_{2}$ and $2 \%$ goat normal serum. Antigen retrieval was achieved by microwaving ( $10 \mathrm{~min}$, $700 \mathrm{~W}$ ) in EDTA buffer, pH 9. The markers eNOS and VEGF were quantitatively assessed by counting the number of visible nuclei per $\mathrm{mm}^{2}$ surface within the subcutaneous tissue.

\section{Test Substance}

Epoetin $\beta$ (recombinant human EPO, NeoRecormon; Roche Pharma, Basel, Switzerland) was admixed to $0.3 \mathrm{ml} \mathrm{NaCl}$ $0.9 \%$ to achieve a final dose of $500 \mathrm{IU}$ per $\mathrm{kg}$ bw. The solution was stored at a maximum temperature of $8^{\circ} \mathrm{C}$ until usage.

$N^{\omega}$-nitro-L-arginine methyl ester (L-Name: SigmaAldrich), the L-arginine analog, which inhibits NOS in a nonspecific manner, was dissolved in sterile saline at room temperature to a final dose of $50 \mathrm{mg}$ per $\mathrm{kg}$ bw and administered slowly i.v.

Bevacizumab (Avastin; Roche Pharma), a monoclonal antibody that acts as a VEGF receptor blocker, was dissolved in sterile saline at room temperature to a final dose of $5 \mathrm{mg}$ per kg bw and administered i.p.

\section{Experimental Groups and Protocol}

A total of 78 animals were equipped with flaps and dorsal skinfold chambers. In all, 42 mice were assigned to 6 experimental groups of 7 animals each: (1) EPO (500 IU per kg bw, i.p.); (2) EPO and L-Name (500 IU EPO per kg bw, i.p. $+50 \mathrm{mg}$ L-Name per $\mathrm{kg}$ bw, i.v.); (3) L-Name (50 mg per $\mathrm{kg}$ bw, i.v.); (4) EPO and bevacizumab (500 IU EPO per kg bw, i.p. $+5 \mathrm{mg}$ bevacizumab per $\mathrm{kg}$ bw, i.p.); (5) Flap (untreated flap: $0.3 \mathrm{ml} \mathrm{NaCl}$, i.p.); and (6) control (nonischemic chamber: $0.3 \mathrm{ml} \mathrm{NaCl}$, i.p.). All animals were administered three repetitive doses of the substance $30 \mathrm{~min}$ before and $30 \mathrm{~min}$ after surgery (that is, induction of ischemia), as well as $24 \mathrm{~h}$ after flap elevation. In case of combined application (EPO and L-Name, and EPO and bevacizumab), L-Name and bevacizumab were administered $5 \mathrm{~min}$ before $\mathrm{EPO}$ at each of the three time points. Intravital fluorescence microscopic analysis of microhemodynamics, apoptotic cell death, macromolecular leakage and angiogenesis was carried out at days $1,3,5,7$ and 10 after surgery. All animals were killed at

Figure 1 Flap tissue morphology at day 10 after elevation shows a clear demarcation zone within the critically perfused central flap area of untreated flaps, including a red fringe (double arrowhead), representing a hyperemic response and microvascular remodeling, delimiting necrosis developing distally (asterisk) (a). Exclusive administration of erythropoietin (EPO) (b) results in increased flap survival, whereas additional administration of L-Name abrogates EPO-induced improvement of flap survival (c). L-Name administration only further worsens flap survival (d). Magnification $\times 16$. Time course of necrosis in untreated flaps (white squares), in animals receiving EPO only (white circles), EPO and L-Name (gray circles), and L-Name only (black circles) (e), as well as EPO and bevacizumab (black triangles). Nonischemic chambers (controls) are represented by white triangles (f). Note that animals administered with EPO only show an improved perfusion already at day 1, resulting in a reduced flap necrosis at day 10 (e), whereas administration of L-Name alone presents an early microcirculatory failure at day 1 with progressive necrosis indicating the abrogation of ischemia-induced protection. Additional administration of bevacizumab does not show any change in flap survival when compared with mice receiving EPO only (f). Mean \pm s.e.m.; ${ }^{*} P<0.05$ vs untreated flaps, ${ }^{\#} P<0.05$ vs controls (nonischemic chambers); $n=7$ per group. 
the end of the experiment by administration of $1 \mathrm{ml}$ of the anesthetic agent. Finally, four additional mice each (EPO, EPO and L-Name, and untreated flap) were killed at days 1, 3 and 5 after induction of ischemia to study the protein profiling of eNOS and VEGF in viable tissue using immunohistochemical analysis.
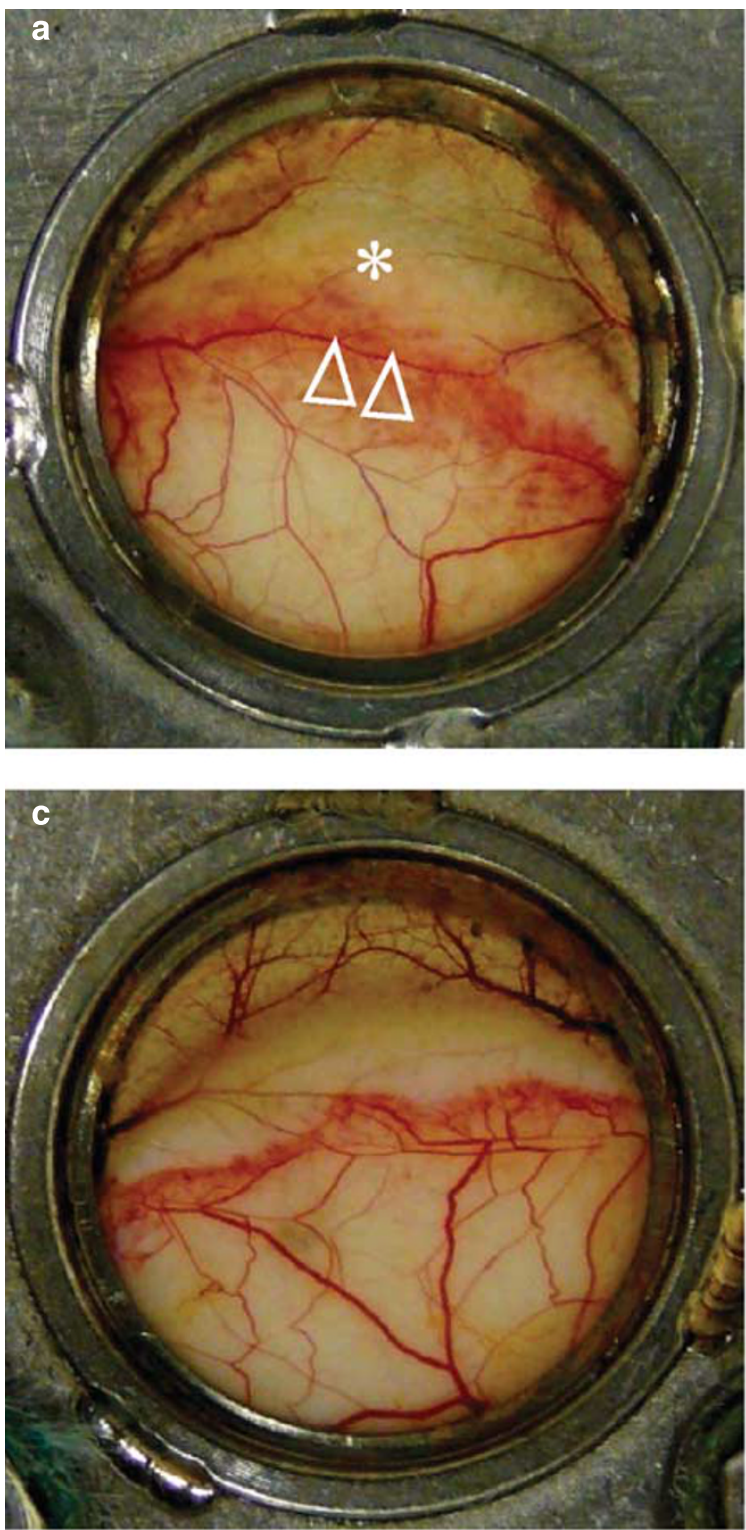

e

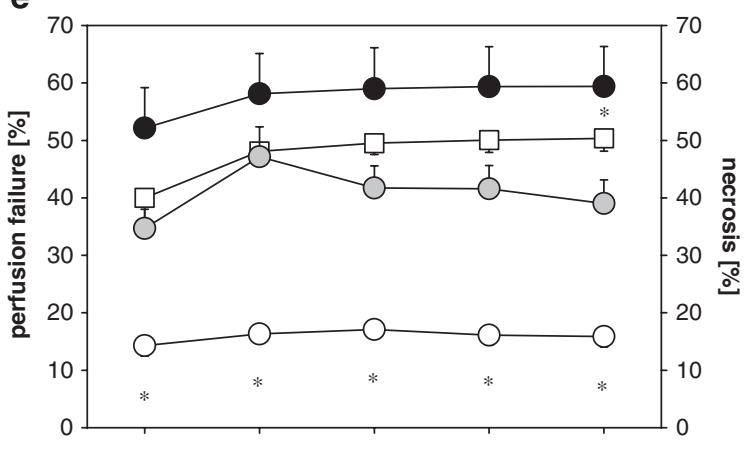

time after flap elevation [d]
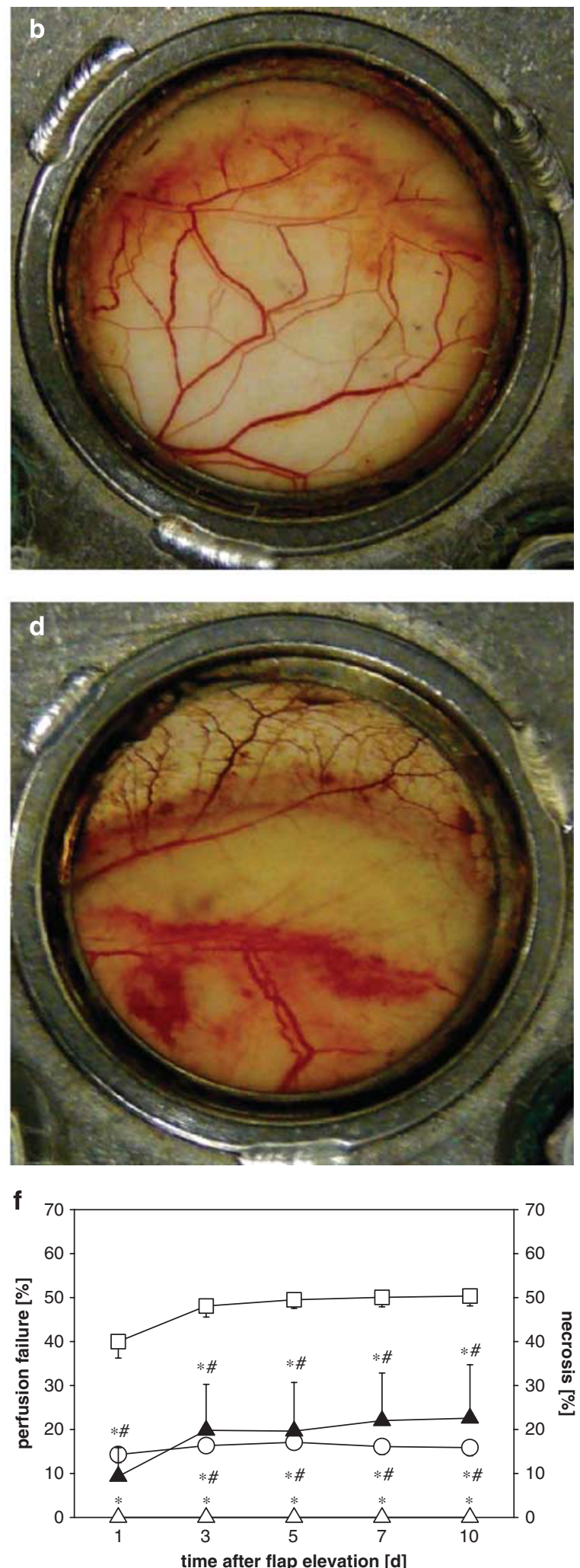
Flap preparation, microscopic examinations, as well as the off-line analysis of the collected data were carried out under blinded conditions.

\section{Statistical Analysis}

All values are expressed as mean \pm s.e.m. For comparison between individual time points, ANOVA for repeated measures was carried out, followed by the appropriate post hoc test (SigmaStat, Jandel, San Rafael, CA, USA), which included measures to correct the $\alpha$-error according to Bonferroni probabilities. Comparison between the groups included ANOVA and the Student Newman-Keuls post hoc test. Differences were considered significant at $P<0.05$.

\section{RESULTS}

\section{Flap Necrosis}

Macroscopic analysis of the chamber morphology at day 10 after surgery showed distinct alterations in the central and distal areas of the flaps (Figures 1a-d). The vital tissue was separated from the distal necrosis by a red fringe indicating vasodilation and microvascular remodeling (Figure 1a). Animals treated with EPO only showed an improved tissue survival (Figure 1b), whereas coadministration of EPO and L-Name did not improve survival (Figure 1c). Of interest, application of L-Name alone was associated with further development of necrosis when compared with controls (Figure 1d).

The initial microcirculatory perfusion failure of $40 \pm 4 \%$ of the chamber surface at day 1 led to necrosis of $50 \pm 2 \%$ at day 10 in animals equipped with untreated flaps. Exclusive EPO administration showed already at day 1 a significantly decreased microcirculatory perfusion failure, which was associated with a reduction of the non-perfused tissue area of $13 \pm 1 \%(P<0.05$ vs untreated flap $)$ and tissue necrosis of $16 \pm 2 \%$ at day $10 \quad(P<0.05)$ (Figure $1 \mathrm{e})$. Microcirculatory dysfunction following coadministration of EPO and L-Name was comparable with that of untreated flaps, whereas application of L-Name alone further deteriorated tissue survival when compared with untreated flaps $(P<0.05)$ (Figure 1e). It is interesting that flaps treated with EPO and bevacizumab did not show any change in tissue necrosis when compared with animals receiving EPO only (Figure 1f). Nonischemic controls, for example, mice undergoing chamber preparation without flap surgery did not develop necrosis (Figure 1f).

\section{Arteriolar Perfusion}

Red blood cell velocity slightly increased in all animals during the 10-day observation period (Figure 2a). Administration of EPO, as well as coadministration of EPO and bevacizumab led to an arteriolar dilation (Figures $2 \mathrm{~b}$ and $\mathrm{c}$ ), whereas all other groups did not show any change in arteriolar diameter, including nonischemic controls (Figures $2 \mathrm{~b}$ and $\mathrm{c}$ ). As a consequence, exclusive EPO administration resulted in a significantly increased arteriolar blood flow already briefly after surgery, which further increased during the observation period when compared with untreated flaps, animals receiving L-Name with or without EPO (Figure 2d) and nonischemic chambers (data not shown).

\section{eNOS Expression}

Administration of EPO led to a threefold increase of eNOS expression at day 1 and a twofold increase at days 3 and 5 after surgery (that is, induction of ischemia) $(P<0.05)$ (Figures $3 \mathrm{~b}$ and $\mathrm{d}$ ), whereas untreated flaps and mice receiving the combination of EPO and L-Name did not show any increase of protein expression when compared with baseline (day 0) (Figures 3a, c and d). Accordingly, diameter augmentation of arteriovenular bundles was observed in animals receiving EPO only (Figure 3d), whereas both untreated flaps and mice administered with L-Name did not develop any noticeable morphological changes in diameter neither in arterioles nor in venules (Figures $3 e$ and $f$ ).

\section{Capillary Perfusion}

Functional capillary density within the proximal and central area of the chamber preparation was significantly reduced already at day 1 after surgery in all animals receiving L-Name (L-Name, EPO and L-Name) $(P<0.05$ vs untreated flaps; Table 1), whereas administration of EPO was associated with a significantly increased number of perfused capillaries. In the distal area, FCD was maintained over the 10-day observation period only in the EPO group, whereas all other groups with flaps revealed complete cessation of FCD from day 3 onwards. It is interesting that FCD within the surviving proximal and central flap area of animals receiving EPO only was comparable with FCD observed in corresponding chamber areas of nonischemic controls (Table 1).

\section{Vascular Remodeling, Angiogenesis and Macromolecular Leakage}

Vascular remodeling with dilation and increased tortuosity of the microvessels was observed in all experimental groups undergoing flap preparation (Figures $4 \mathrm{a}, \mathrm{b}, \mathrm{d}$ and e). Administration of EPO induced angiogenesis, which was completely abrogated by additional administration of bevacizumab (Figure 4i). The new functional microvascular networks that usually develop from microvascular buds and sprouts were first visible at day 5 (Figures $4 \mathrm{~b}, \mathrm{c}$ and g). Angiogenesis was observed in all animals receiving EPO, regardless of coadministration of L-Name (Figures $4 b$ and c), but not in untreated flaps or in mice receiving L-Name only (Figure 4a, d, g and i). Significant macromolecular leakage, that is, microvascular permeability of capillaries was observed from day 1 onwards and persisted over the entire 10-day observation period in all experimental groups undergoing treatment, that is, EPO, EPO and L-Name, and L-Name. Microvascular permeability was particularly increased if EPO was administered, whereas untreated flaps showed less microvascular leakage, but considerably more than nonischemic chambers (Figures $5 \mathrm{a}$ and $\mathrm{b}$; Table 2). 

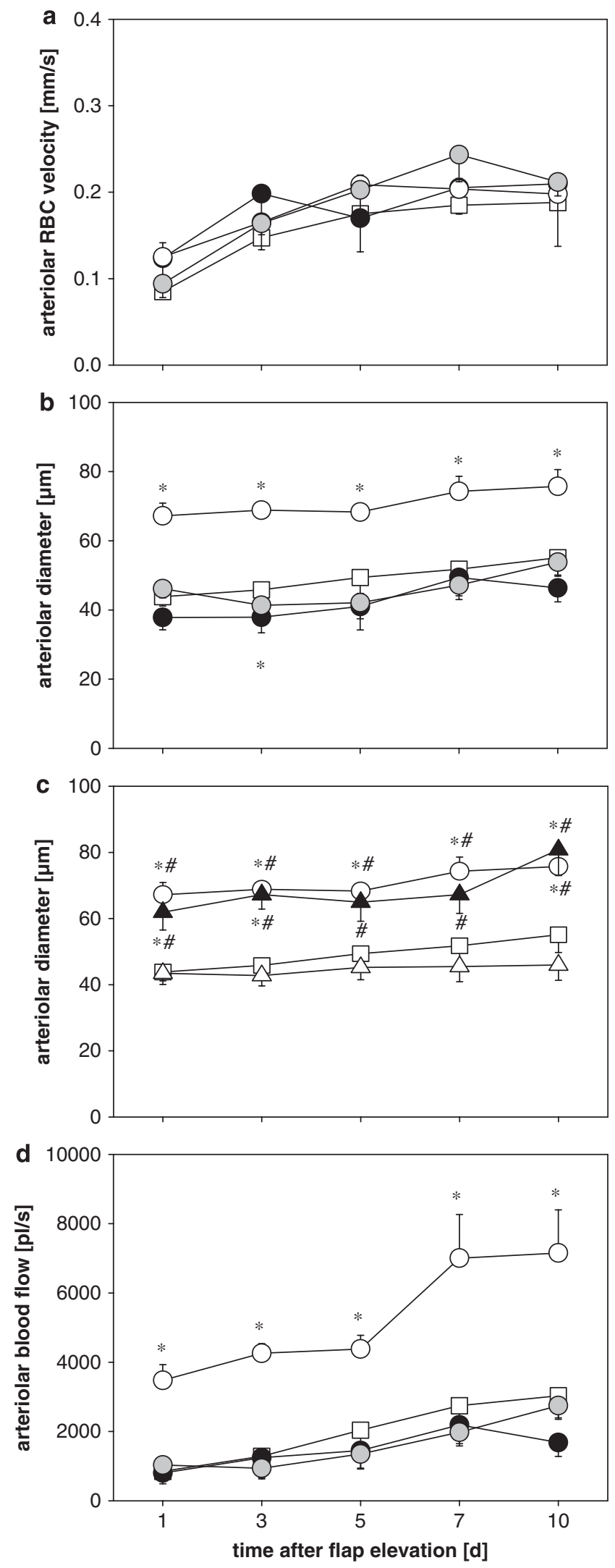

\section{VEGF Expression}

The expression of VEGF was time dependent in both groups (untreated flaps and EPO). Although the baseline expression of VEGF was comparable in untreated flaps and mice administered with EPO only, the increased expression at day 3 after surgery was more pronounced and became significant from day 5 onwards in animals administered with EPO (Figure 4h).

\section{Apoptotic Cell Death}

Induction of persistent ischemia after acute injury in untreated flaps and animals receiving L-Name only was associated with a considerable number of apoptotic cell death at days 1,3 and 5 showing one of the characteristic signs, that is nuclear condensation (Figures $6 \mathrm{a}, \mathrm{b}$ and e). EPO administration significantly decreased cellular apoptosis to a level that was comparable with the surgery-induced apoptotic cell death observed in nonischemic chambers also, if L-Name was coadministered with EPO $(P<0.05 v s$ untreated flap).

\section{DISCUSSION}

This study shows that administration of EPO before and after induction of ischemia in musculocutaneous tissue subjected to persistent ischemia is capable of attenuating ischemia-induced tissue injury by modulating microcirculatory properties. This protective effect seems to be predominantly caused by microvascular dilation mediated by the NO-releasing enzyme eNOS. This guarantees sufficient capillary perfusion of the tissue jeopardized by ischemia and results in a significant reduction of tissue necrosis. VEGF-associated formation of new functional capillaries does not seem to have a role in EPO-mediated tissue protection, whereas prevention of apoptotic cell death might occur for some protection in this model.

Induction of ischemia, that is, surgical preparation, caused a microvascular breakdown that resulted in tissue necrosis of $\sim 50 \%$ at day 10 in untreated animals. Three administrations of recombinant human EPO starting at 30 min before surgery significantly improved tissue survival. This was the consequence of an increased dilation of feeding arterioles mediated by an early upregulation of eNOS, which resulted in sustained capillary perfusion. Coadministration of the unspecific NOS antagonist L-Name completely overruled this dilatory response in arterioles and annihilated EPO-mediated tissue protection. This is in line with a previous study that showed that EPO induced basal vasodilation in $\mathrm{rHuEPO}$ hypertensive rats in an eNOS-dependent manner. ${ }^{25}$

Figure 2 Red blood cell velocity (a), diameter (b, c) and blood flow (d) in arterioles over the 10-day observation period within the critically perfused tissue in untreated flaps (white squares), in animals receiving erythropoietin (EPO) only (white circles), EPO and L-Name (gray circles), and L-Name only (black circles), as well as EPO and bevacizumab (black triangles). Nonischemic chambers (controls) are represented by white triangles. Mean \pm s.e.m.; ${ }^{\star} P<0.05$ vs untreated flaps, ${ }^{\#} P<0.05$ vs nonischemic chambers (controls); $n=7$ per group. 

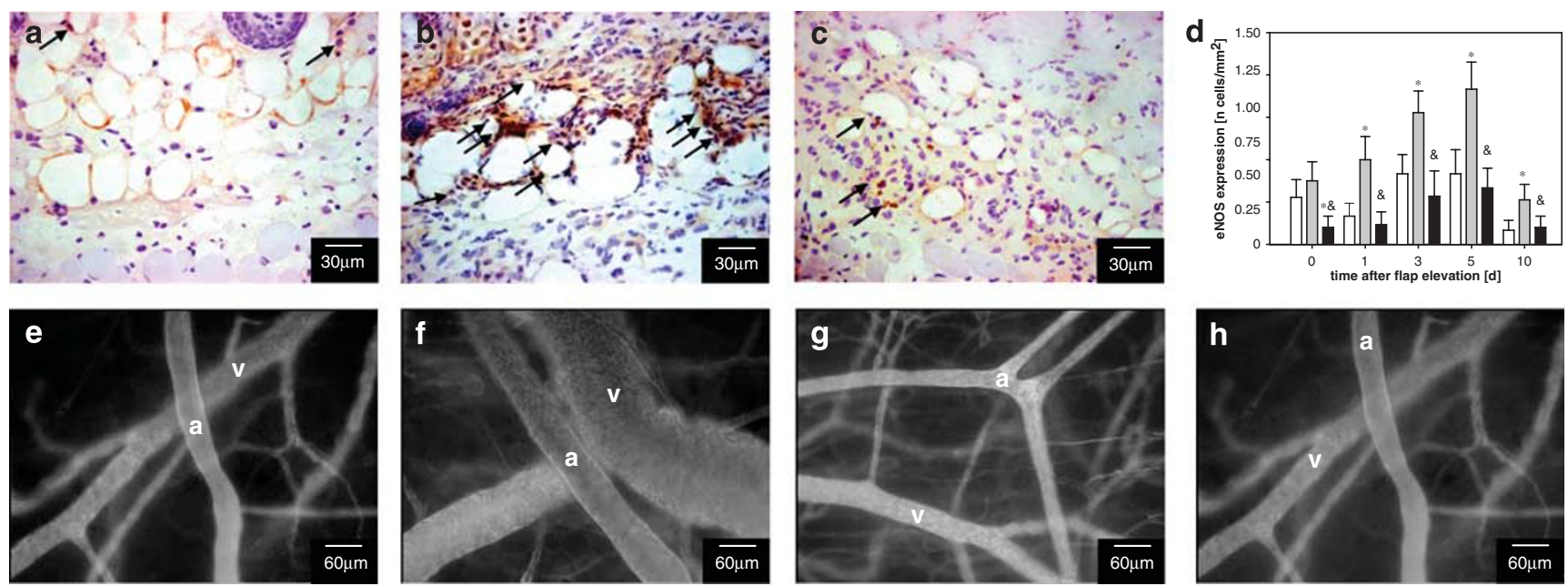

Figure 3 Longitudinal sections of viable tissue 5 days after induction of ischemia stained for endothelial nitric oxide synthase (eNOS) expression in untreated flaps (a), animals receiving erythropoietin (EPO) only (b), as well as in animals receiving a coadministration of EPO and L-Name (c). Note the nuclear and cytosolic staining predominantly within the subcutaneous tissue of EPO-administered flaps (arrows). Magnification $\times 160$. Quantitative analysis of the 10-day time course of eNOS expression in untreated flaps (white bars), animals receiving EPO only (gray bars) or EPO and L-Name (black bars). Note the threefold increase of eNOS expression at day 1 and the twofold increase between days 3 and 5 after surgery after EPO administration only (d). Mean \pm s.e.m.; ${ }^{*} P<0.05$ vs untreated flaps, ${ }^{\&} P<0.05$ vs EPO; $n=4$ per group. Intravital epifluorescence microscopy displaying arteriolovenular bundles at day 1 in an untreated flap (e), as well as in an animal administered with EPO only (f), EPO and L-Name (g) or L-Name only (h). Note the lack of vascular dilation after administration of L-Name ( $\mathbf{g}, \mathbf{h})$ when compared with EPO administration displaying a considerable increase of arteriolar (a) and venular (v) diameter (f). Contrast enhancement with fluorescein isothiocyanate (FITC)-dextran 150000 . Magnification $\times 80$.

Table 1 Functional capillary density $\left(\mathrm{cm} / \mathrm{cm}^{2}\right)$ within the proximal, central and distal area of the flap at days 1, 3, 5, 7 and 10 after induction of ischemia

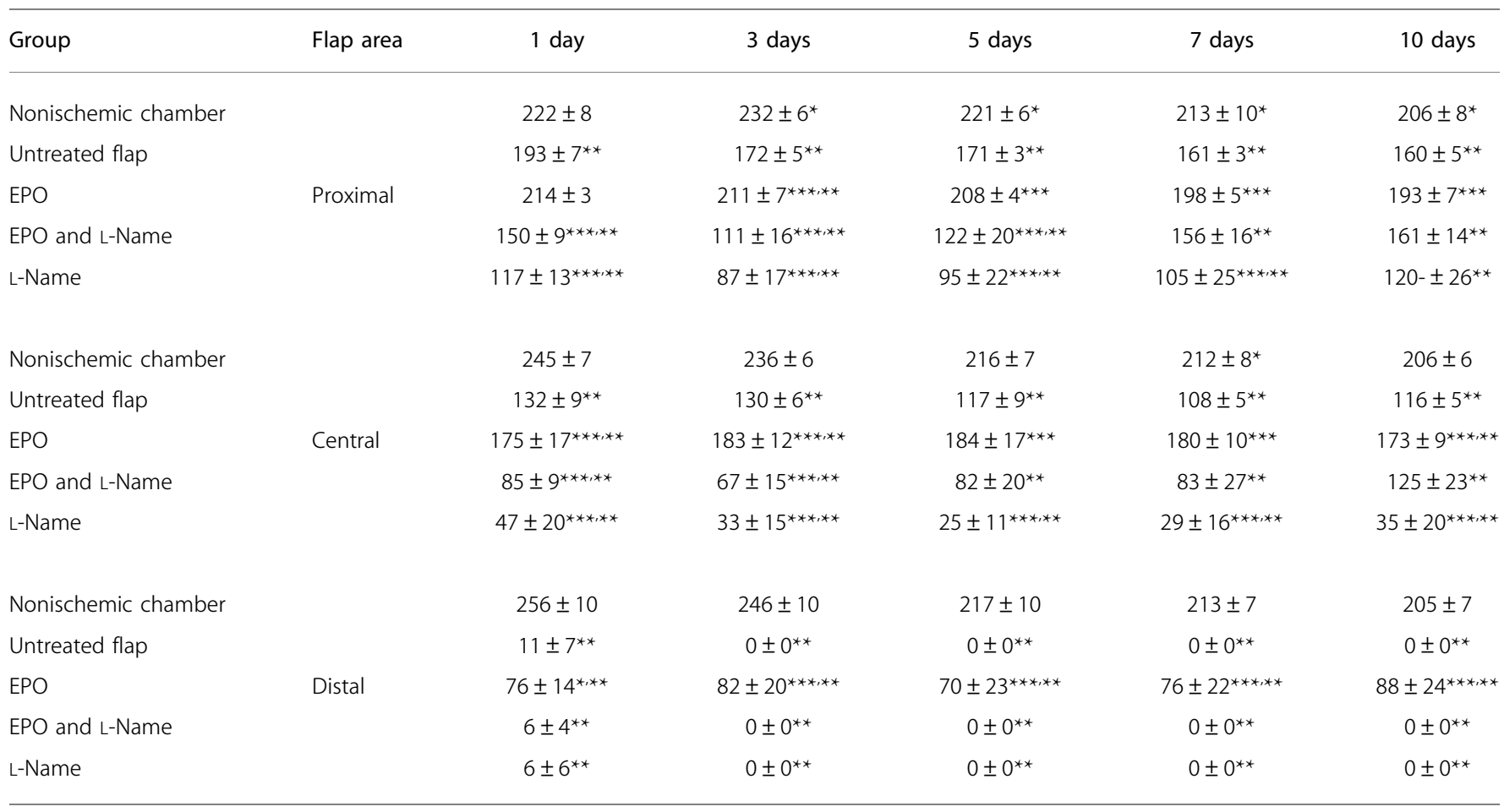

Values are mean \pm s.e.m.

${ }^{*} P<0.05$ vs day 1 (in controls), ${ }^{* *} P<0.05$ vs nonischemic chambers (controls), ${ }^{* *} P<0.05$ vs untreated flaps. 

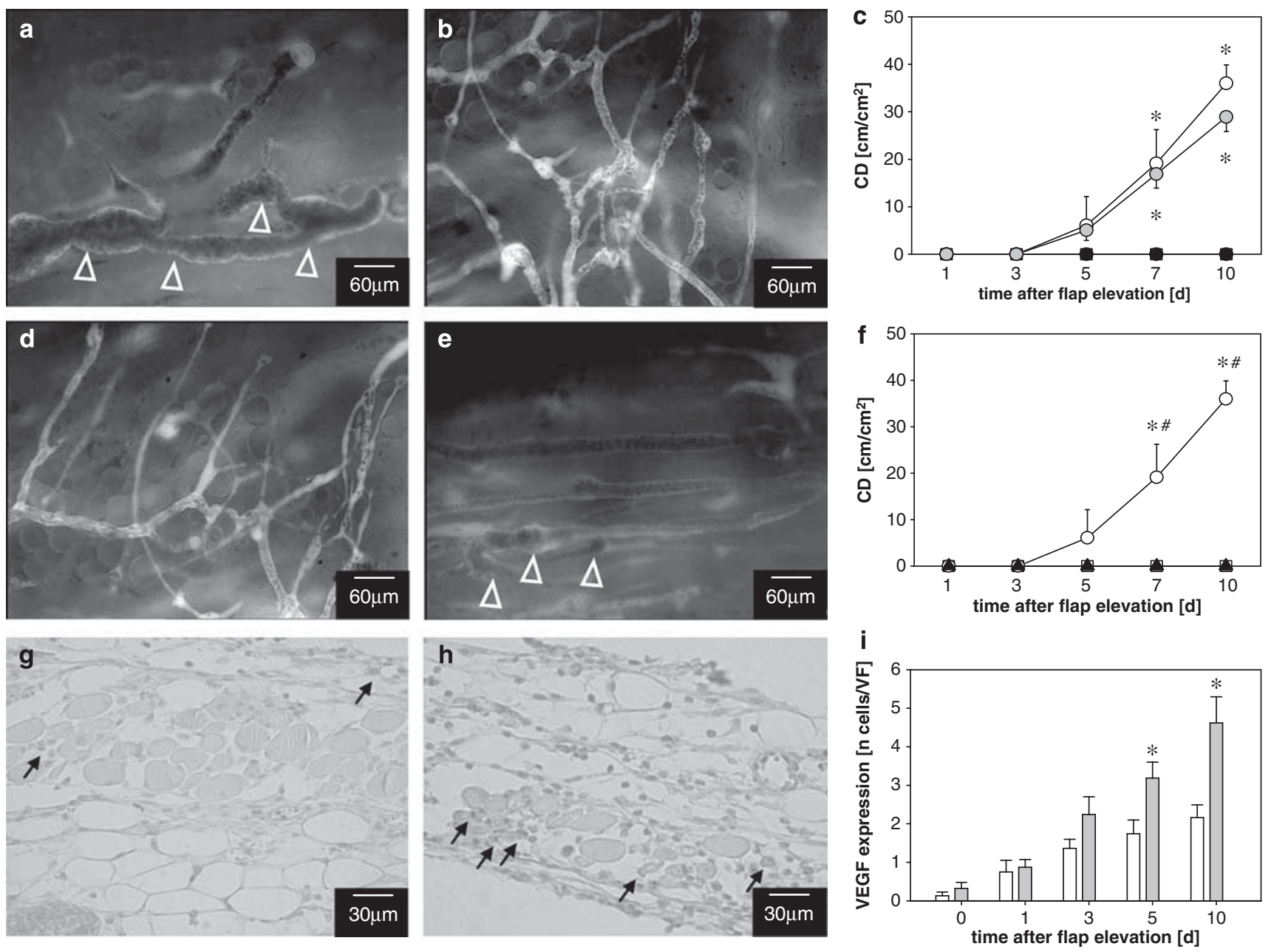

Figure 4 Intravital epifluorescence microscopy of microvascular remodeling in untreated flaps (a) and animals receiving L-Name only (e) characterized by dilation, increased tortuosity of in-parallel arranged striated muscle capillaries, as well as buds and sprouts (arrow heads). Note the formation of new functional microvessels perpendicular to the preexisting capillary network observed 10 days after flap elevation only after administration of erythropoietin (EPO) only (b), with regard to coadministration of EPO and L-Name (d). Contrast enhancement with fluorescein isothiocyanate (FITC)-dextran 150000. Magnification $\times 80$. Longitudinal sections of viable tissue 10 days after induction of ischemia stained for vascular endothelial growth factor (VEGF) expression in untreated flaps $(\mathbf{g})$ and animals receiving EPO only (h). Note the nuclear and cytosolic staining predominantly within the subcutaneous and muscular tissue of EPO-administered flaps (h; arrows). Magnification $\times 160$. Quantitative analysis of the density of newly formed microvessels in untreated flaps (white squares), in animals receiving EPO only (white circles), EPO and L-Name (gray circles), and L-Name only (black circles) (c), as well as EPO and bevacizumab (black triangles). Nonischemic chambers are represented by white triangles (f) over the 10-day observation period within the critically perfused flap area. Note the significant angiogenic response in animals receiving EPO that is completely abrogated after bevacizumab administration (i) ( $n=7 /$ group). Quantitative analysis of the 10-day time course of VEGF expression in untreated flaps (white bars) and in animals receiving EPO only (gray bars). Mean \pm s.e.m.; ${ }^{*} P<0.05$ vs untreated flaps, ${ }^{\#} P<0.05$ vs nonischemic chambers (controls); $n=4$ per group.

This protective effect was completely abolished after additional administration of L-Name increasing blood pressure and total peripheral resistance. In muscle tissue it was shown that a single dose of $5000 \mathrm{IU}$ EPO per kg bw, notably, a $10 \times$ higher dose than the one used in this study, was able to attenuate ischemia/reperfusion injury by increasing NO bioavailability mediated by upregulation of eNOS. ${ }^{10}$ Further, this single dose of EPO administered before induction of ischemia has shown to induce eNOS, which was associated with a significantly attenuated ischemia-mediated capillary perfusion failure in musculocutaneous tissue despite an absence of arteriolar dilation. The fact that microscopic observation was limited to $5 \mathrm{~h}$, no conclusion concerning tissue survival could be drawn. ${ }^{25}$ In addition, EPO was capable of preventing hypertension by inducing a dilatory effect through upregulation of the vascular expression of eNOS in rats. ${ }^{26}$ In this study, exclusive administration of L-Name actually increased tissue necrosis by about $10 \%$ to $\sim 60 \%$, indicating an abrogation of ischemia-induced NO supply, that is, endogenous protection.

Erythropoietin has an important role in the induction of post-ischemic VEGF-mediated angiogenesis, as seen in 

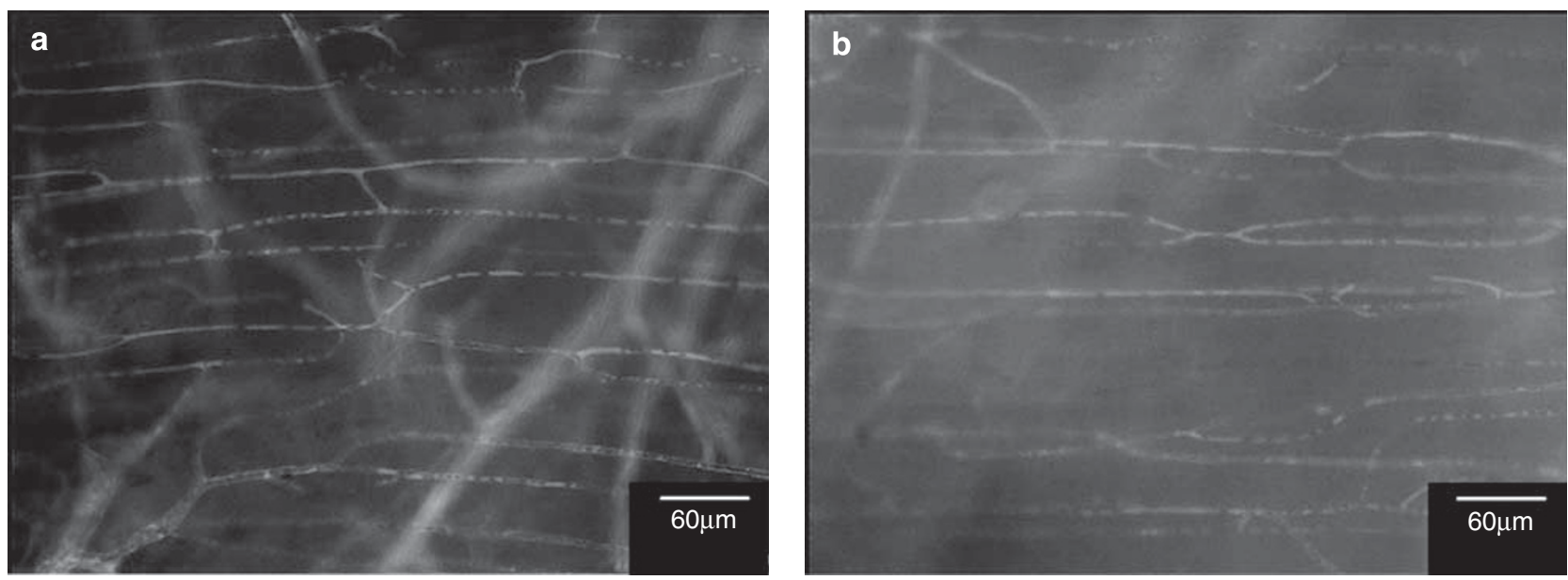

Figure 5 In-parallel arranged capillaries of a nonischemic chamber displaying a clear black and white contrast between interstitial space and intravascular space (a). Loss of contrast between interstitial space and intracapillary space resulting from an increased extravasation of fluorescent contrast media in an animal treated with erythropoietin (EPO) only (b).

Table 2 Macromolecular leakage within the critically perfused central area of the flap at days 1, 3, 5, 7 and 10 after induction of ischemia

\begin{tabular}{|c|c|c|c|c|c|c|}
\hline Group & Flap area & 1 day & 3 days & 5 days & 7 days & 10 days \\
\hline Nonischemic chamber & & $0.58 \pm 0.03$ & $0.58 \pm 0.01$ & $0.63 \pm 0.03$ & $0.59 \pm 0.03$ & $0.68 \pm 0.03$ \\
\hline EPO & Central & $0.84 \pm 0.02^{*}$ & $0.85 \pm 0.01^{* * * *}$ & $0.83 \pm 0.02^{* *, *}$ & $0.84 \pm 0.02^{* *, *}$ & $0.85 \pm 0.02^{* * * *}$ \\
\hline EPO and L-Name & & $0.89 \pm 0.02^{* *, *}$ & $0.85 \pm 0.03^{* *, *}$ & $0.83 \pm 0.02^{* *, *}$ & $0.87 \pm 0.02^{* *, *}$ & $0.85 \pm 0.01^{* * * *}$ \\
\hline
\end{tabular}

Values are mean \pm s.e.m.

${ }^{*} P<0.05$ vs nonischemic chambers (controls), ${ }^{*} P<0.05$ vs untreated flaps.

the ischemic muscle. ${ }^{27,28}$ An improvement of myocardial remodeling and function following EPO treatment after infarction was shown, ${ }^{28}$ and a crucial role for the vascular EPO receptor system in angiogenesis in response to critical hindlimb ischemia mediated through an upregulation of VEGF/VEGF receptor system was shown. ${ }^{27}$ Moreover, EPO was able to stimulate normal endothelial progenitor cellmediated endothelial turnover, but the effect on cardiac microvascularization and function was only seen in the presence of ischemia. ${ }^{29}$ This is in line with our results that show a formation of new microvessels in critically ischemic tissue in all animals with administered EPO independently from coadministration of L-Name, which suggests an EPOmediated angiogenic response under hypoxic conditions. Animals that simultaneously received EPO and bevacizumab showed a complete abrogation of the EPO-induced angiogenic response without affecting the EPO-mediated tissue protection. This indicates the following: (1) The formation of new functional capillary networks is VEGF driven, as the
VEGF antibody bevacizumab prevents from the angiogenic response. (2) The angiogenic response is not involved in EPO-mediated survival of musculocutaneous tissue, most probably because the development of microvessels occurs from days 5-7 onwards. This is in contrast with Galeano et $a l^{30}$ who demonstrated in a murine model of thermal injury that EPO-mediated angiogenesis resulted in significantly improved wound healing if the drug was administered on a daily base for a time period of 14 days. Interestingly, ischemia alone without the administration of EPO (untreated flaps and L-Name) was not able to induce angiogenesis, which indicates that the new functional microvessels in this model only develop in the presence of EPO.

Macromolecular leakage increased significantly after the administration of EPO, as well as L-Name. This may be the result of an overexpression of VEGF-mediating hyperpermeability of microvessels with regard to ischemiainduced microvascular injury. VEGF has previously been 

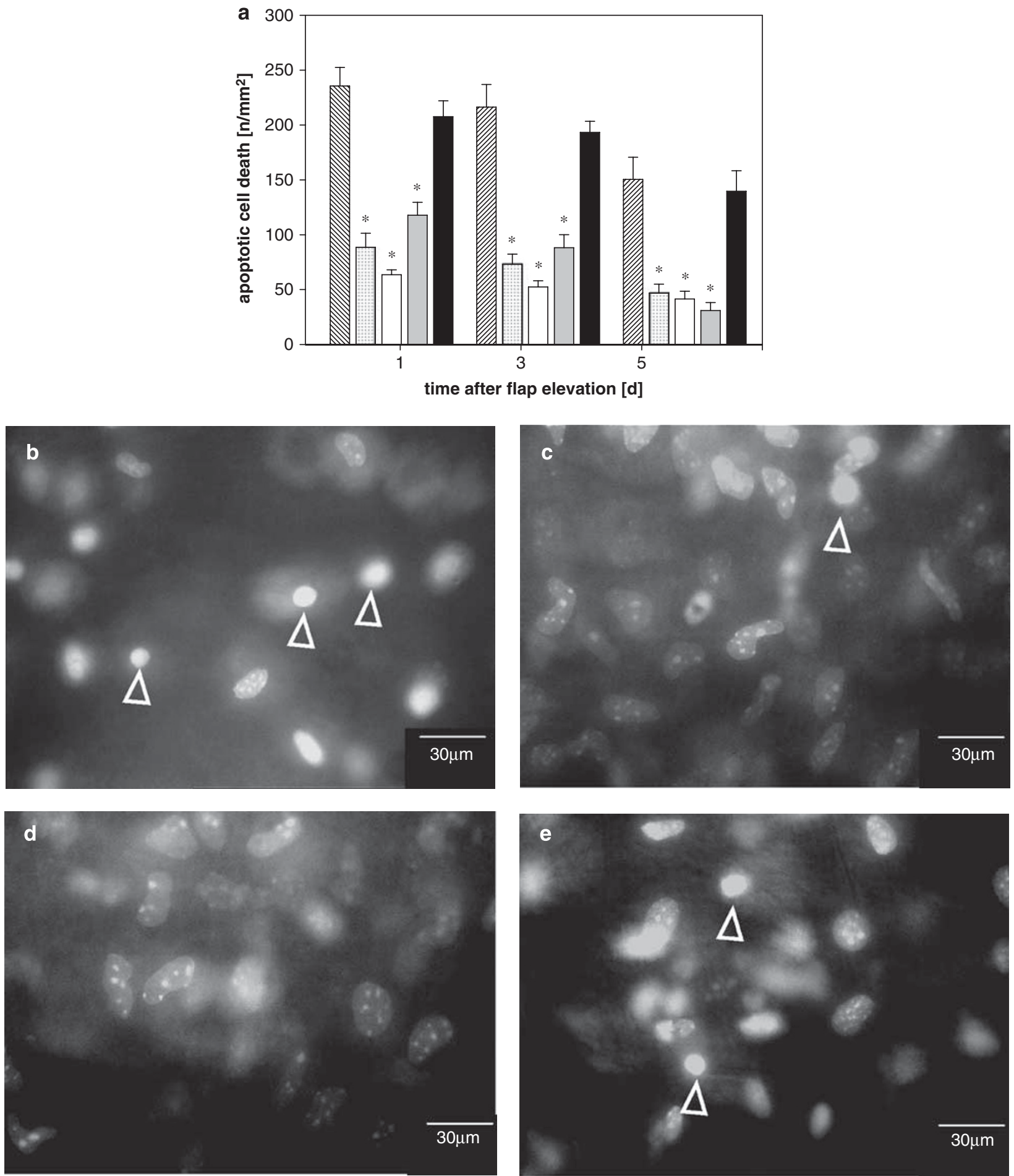

Figure 6 Quantitative analysis of apoptotic cell death within the critically perfused flap area of untreated flaps (hatched bars), as well as in animals receiving erythropoietin (EPO) only (white bars), EPO and L-Name (gray bars) and L-Name only (black bars) at days 1, 3 and 5 after flap elevation. Nonischemic controls are represented by dotted bars (a). Typical view by intravital epifluorescence microscopy in an untreated flap (b), as well as in an animal administered with EPO only (c), EPO and L-Name (d) or L-Name only (e) displaying apoptotic cells showing nuclear condensation (white arrowheads). Note the significant decrease of apoptotic cell death if EPO is administered. Mean \pm s.e.m.; ${ }^{*}<0.05$ vs controls; $n=7$ per group.

shown to induce vascular hyperpermeability in therapeutic angiogenesis. ${ }^{31}$ Further, significant macromolecular leakage has also been associated with increased VEGF protein content and mRNA concentration in hypoxic brain tissue. ${ }^{32}$ In addition, enhanced albumin leakage has been observed in post-capillary venules of the mesentery ${ }^{33}$ and visceral 
organs, ${ }^{34}$ after the administration of L-Name, which indicates that endogenous NO may contribute to microvascular integrity during surgery and ischemia-induced tissue injury.

Several studies have attested EPO anti-apoptotic properties in traumatic, ischemic and toxic injury in a multitude of organs, such as the skeletal muscle, ${ }^{35}$ liver $^{36}$ and kidney. ${ }^{11}$ Our results showed that administration of EPO significantly decreased the number of apoptotic cells when compared with untreated flaps and animals receiving L-Name only. This is in line with a previous study of our group, in which we could show that apoptotic cell death was significantly reduced even if EPO was administered only after flap elevation. Tissue survival, however, was not affected when compared with untreated flaps, suggesting that EPO-mediated reduction of apoptotic cell death was not sufficient to protect ischemically challenged tissue if microvascular perfusion was not maintained, which was the case in animals receiving EPO only after surgery. ${ }^{37}$ These experiments show that a triplicate coadministration of EPO and L-Name before and after surgery is able to counteract tissue necrosis mediated by L-Name by $\sim 20 \%$, in spite of comparable microvascular parameters, that is, arteriolar dilation and arteriolar blood flow. This indicates that reduction of apoptotic cell death mediated by EPO may account for some protection that is not dependent on microvascular blood flow. This observation is in line with EPO administration that reduced apoptotic cell death and recovery of the impaired microcirculation following ischemia-reperfusion injury of the intestine, ${ }^{38}$ liver ${ }^{36}$ and testes. ${ }^{39}$ This indicates that a reduced apoptotic cell death contributing to final tissue survival is most probably dependent on ischemia followed by reperfusion.

In conclusion, this study shows that a triplicate administration of $500 \mathrm{IU}$ per $\mathrm{kg}$ bw EPO started before surgery, that is, induction of ischemia prevents musculocutaneous tissue from necrosis. This can be attributed to an eNOS-dependent vasodilatory response in the arterioles, which maintains the capillary perfusion of the ischemically jeopardized tissue. The protection provided by EPO is almost completely abrogated with concomitant application of L-Name. However, coadministration of EPO and bevacizumab does not affect tissue survival despite complete inhibition of angiogenesis, which suggests that the EPO-mediated angiogenic response is not effective for tissue survival. EPO-mediated anti-apoptotic properties may contribute to tissue survival as long as EPO is administered before induction of ischemia. Altogether, EPO seems to be an up-and-coming approach to pharmacologically influence ischemically challenged tissue.

\section{ACKNOWLEDGEMENTS}

The authors thank Janine Becker (Institute for Clinical \& Experimental Surgery, University of Saarland, D-66421 Homburg/Saar, Germany) for excellent technical assistance, and Anton Haselbeck (Roche Diagnostics GmbH, Penzberg, Germany) and Pierre-Alain Delley (Roche Pharma Schweiz, Reinach, Switzerland) for the drug supply. This study was financially supported by the Swiss National Science Foundation (SNF-No. 3200BO108408).

\section{DISCLOSURE/CONFLICT OF INTEREST}

The authors declare no conflict of interest.

1. Krantz SB. Erythropoietin. Blood 1991;77:419-434.

2. Wu H, Liu X, Jaenisch R, et al. Generation of committed erythroid BFU-E and CFU-E progenitors does not require erythropoietin or the erythropoietin receptor. Cell 1995;83:59-67.

3. Lacombe C, Da Silva JL, Bruneval P, et al. Erythropoietin: sites of synthesis and regulation of secretion. Am J Kidney Dis 1991;18 (4 Suppl 1):14-19.

4. Hasegawa J, Wagner KF, Karp D, et al. Altered pulmonary vascular reactivity in mice with excessive erythrocytosis. Am J Respir Crit Care Med 2004;169:829-835.

5. Cai Z, Semenza GL. Phosphatidylinositol-3-kinase signaling is required for erythropoietin-mediated acute protection against myocardial ischemia/reperfusion injury. Circulation 2004;109:2050-2053.

6. Sakanaka M, Wen TC, Matsuda S, et al. In vivo evidence that erythropoietin protects neurons from ischemic damage. Proc Natl Acad Sci USA 1998;95:4635-4640.

7. Hasselblatt $M$, Ehrenreich $H$, Siren AL. The brain erythropoietin system and its potential for therapeutic exploitation in brain disease. J Neurosurg Anesthesiol 2006;18:132-138.

8. Sepodes B, Maio R, Pinto R, et al. Recombinant human erythropoietin protects the liver from hepatic ischemia-reperfusion injury in the rat. Transpl Int 2006;19:919-926.

9. Patel NS, Sharples EJ, Cuzzocrea S, et al. Pretreatment with EPO reduces the injury and dysfunction caused by ischemia/reperfusion in the mouse kidney in vivo. Kidney Int 2004;66:983-989.

10. Contaldo C, Meier C, Elsherbiny A, et al. Human recombinant erythropoietin protects the striated muscle microcirculation of the dorsal skinfold from postischemic injury in mice. Am J Physiol Heart Circ Physiol 2007;293:H274-H283.

11. Salahudeen AK, Haider N, Jenkins J, et al. Anti-apoptotic properties of erythropoiesis stimulating proteins in models of cisplatin-induced acute kidney injury. Am J Physiol Renal Physiol 2008;294:F1354-F1365.

12. Xue $Y Q$, Zhao LR, Guo WP, et al. Intrastriatal administration of erythropoietin protects dopaminergic neurons and improves neurobehavioral outcome in a rat model of Parkinson's disease. Neuroscience 2007;146:1245-1258.

13. Burger D, Lei M, Geoghegan-Morphet $\mathrm{N}$, et al. Erythropoietin protects cardiomyocytes from apoptosis via up-regulation of endothelial nitric oxide synthase. Cardiovasc Res 2006;72:51-59.

14. Katsuki S, Murad F. Regulation of adenosine cyclic $3^{\prime}, 5^{\prime}-$ monophosphate and guanosine cyclic $3^{\prime}, 5^{\prime}$-monophosphate levels and contractility in bovine tracheal smooth muscle. Mol Pharmacol 1977;13:330-341.

15. Bodo E, Kromminga A, Funk W, et al. Human hair follicles are an extrarenal source and a nonhematopoietic target of erythropoietin. FASEB J 2007;21:3346-3354.

16. Stewart KJ, Stewart DA, Coghlan B, et al. Complications of 278 consecutive abdominoplasties. J Plast Reconstr Aesthet Surg 2006;59:1152-1155.

17. Wettstein R, Schürch R, Banic A, et al. Review of 197 consecutive free flap reconstructions in the lower extremity. J Plast Reconstr Aesthet Surg 2008;61:772-776.

18. Ascherman JA, Patel SM, Malhotra SM, et al. Management of sternal wounds with bilateral pectoralis major myocutaneous advancement flaps in 114 consecutively treated patients: refinements in technique and outcomes analysis. Plast Reconstr Surg 2004;114:676-683.

19. Zeitani J, Bertoldo F, Bassano C, et al. Superficial wound dehiscence after median sternotomy: surgical treatment versus secondary wound healing. Ann Thorac Surg 2004;77:672-675.

20. Lehr HA, Leunig M, Menger MD, et al. Dorsal skinfold chamber technique for intravital microscopy in nude mice. Am J Pathol 1993;143:1055-1062.

21. Harder $Y$, Amon M, Erni D, et al. Evolution of ischemic tissue injury in a random pattern flap: a new mouse model using intravital microscopy. J Surg Res 2004;121:197-205.

22. Klyscz T, Junger M, Jung F, et al. [Cap image-a new kind of computerassisted video image analysis system for dynamic capillary microscopy]. Biomed Tech (Berl) 1997;42:168-175. 
23. Gross JF, Aroesty J. Mathematical models of capillary flow: a critical review. Biorheology 1972;9:225-264.

24. Menger MD, Pelikan S, Steiner D, et al. Microvascular ischemiareperfusion injury in striated muscle: significance of 'reflow paradox'. Am J Physiol 1992;263(6 Part 2):H1901-H1906.

25. Contaldo C, Elsherbiny A, Lindenblatt N, et al. Erythropoietin enhances oxygenation in critically perfused tissue through modulation of nitric oxide synthase. Shock 2009;31:599-606.

26. Kanagy NL, Perrine MF, Cheung DK, et al. Erythropoietin administration in vivo increases vascular nitric oxide synthase expression. J Cardiovasc Pharmacol 2003;42:527-533.

27. Nakano M, Satoh K, Fukumoto $Y$, et al. Important role of erythropoietin receptor to promote VEGF expression and angiogenesis in peripheral ischemia in mice. Circ Res 2007;100:662-669.

28. Kobayashi H, Minatoguchi S, Yasuda S, et al. Post-infarct treatment with an erythropoietin-gelatin hydrogel drug delivery system for cardiac repair. Cardiovasc Res 2008;79:611-620.

29. Westenbrink BD, Oeseburg H, Kleijn L, et al. Erythropoietin stimulates normal endothelial progenitor cell-mediated endothelial turnover, but attributes to neovascularization only in the presence of local ischemia. Cardiovasc Drugs Ther 2008;22:265-274.

30. Galeano M, Altavilla D, Bitto A, et al. Recombinant human erythropoietin improves angiogenesis and wound healing in experimental burn wounds. Crit Care Med 2006;34:1139-1146.

31. Sano $H$, Hosokawa $K$, Kidoya $H$, et al. Negative regulation of VEGF-induced vascular leakage by blockade of angiotensin II type 1 receptor. Arterioscler Thromb Vasc Biol 2006;26:

2673-2680.

32. Schoch HJ, Fischer S, Marti HH. Hypoxia-induced vascular endothelial growth factor expression causes vascular leakage in the brain. Brain 2002;125(Part 11):2549-2557.

33. Kurose I, Wolf R, Grisham MB, et al. Effects of an endogenous inhibitor of nitric oxide synthesis on postcapillary venules. Am J Physiol 1995;268(6 Part 2):H2224-H2231.

34. Laszlo F, Morschl E, Pavo I, et al. Nitric oxide modulates the gastrointestinal plasma extravasation following intraabdominal surgical manipulation in rats. Eur J Pharmacol 1999;375:211-215.

35. Rotter R, Menshykova M, Winkler T, et al. Erythropoietin improves functional and histological recovery of traumatized skeletal muscle tissue. J Orthop Res 2008;26:1618-1626.

36. Le Minh K, Klemm K, Abshagen K, et al. Attenuation of inflammation and apoptosis by pre- and posttreatment of darbepoetinalpha in acute liver failure of mice. Am J Pathol 2007;170: 1954-1963.

37. Rezaeian F, Wettstein R, Amon M, et al. Erythropoietin protects critically perfused flap tissue. Ann Surg 2008;248:919-929.

38. Mori S, Sawada T, Okada T, et al. Erythropoietin and its derivative protect the intestine from severe ischemia/reperfusion injury in the rat. Surgery 2008;143:556-565.

39. Ergur BU, Kiray M, Pekcetin C, et al. Protective effect of erythropoietin pretreatment in testicular ischemia-reperfusion injury in rats. J Pediatr Surg 2008;43:722-728. 